\title{
CYCLIC ELEMENTS IN SOME SPACES OF ANALYTIC FUNCTIONS
}

\author{
BY BORIS KORENBLUM ${ }^{1}$
}

Definitions. 1. $A^{-p}(p>0)$ is the Banach space of analytic functions $f(z)$ in $U=\{z \in \mathrm{C}|| z \mid<1\}$ that satisfy $|f(z)|=o\left[(1-|z|)^{-p}\right](|z| \rightarrow 1)$ with the norm $\|f\|=\max \left\{|f(z)|(1-z)^{p}\right\} \quad(z \in U)$. Note that $f_{n} \rightarrow f$ in $A^{-s}$ and $g_{n} \rightarrow g$ in $A^{-t}$ implies $f_{n} g_{n} \rightarrow f g$ in $A^{-(s+t)}$.

2. $B^{p}(p>0)$ is the Bergman space, i.e., the "analytic" subspace of $L^{p}(r d r d \theta)$ in $U$.

3. $A^{-\infty}=\bigcup A^{-p}=\bigcup B^{p}(p>0) . A^{-\infty}$ is a linear topological space [1].

4. $P$ is the set of all algebraic polynomials $P(z)$. $P$ is dense in any of the spaces $A^{-p}, B^{p}, A^{-\infty}$.

5. Let $A$ be any of the spaces $A^{-p}, B^{p}, A^{-\infty}$ and let $f \in A$. The ideal generated by $f$ in $A$ is defined by

$$
I(f ; A)=\operatorname{clos}\{f P \mid P \in P\} .
$$

If $f$ is bounded, then also $I(f ; A)=\operatorname{clos}\{f g \mid g \in A\}$.

6. An $f \in A$ is called cyclic in $A$ if $I(f ; A)=A$.

7. A closed set $E \subset \partial U$ is called a Carleson set if its Lebesgue measure $|E|=0$ and $\Sigma_{n}\left|I_{n}\right| \log \left(2 \pi /\left|I_{n}\right|\right)<\infty$, where $I_{n}$ are the components of $\partial U \backslash E$.

THEOREM. A singular inner function

$$
s(z)=\exp \left\{-\frac{\zeta+z}{\zeta-z} d v(\zeta)\right\},
$$

where $v$ is a nonnegative singular measure on $\partial U$, is cyclic in any (and hence in all) of the spaces $A^{-\infty}, A^{-p}, B^{p}$ if and only if $v(E)=0$ for all Carleson sets $E$.

The "only if" part is due to H. S. Shapiro [2]. The case $A^{-\infty}$ was treated in [3]. Some partial results in a different direction are due to Daniel H. Luecking.

Since every $A^{-p}$ is a dense subset of some $B^{p^{\prime}}$, and vice versa, it suffices to prove the Theorem for $A^{-p}$. Now we use the following result from [3]; it is, roughly, equivalent to the above Theorem for $A^{-\infty}$.

Received by the editors March 18, 1981.

1980 Mathematics Subject Classification. Primary $30 \mathrm{H05.}$

${ }^{1}$ This research was supported by NSF grants MCS78-01878 and MCS80-03413.

(c) 1981 American Mathematical Society $0002-9904 / 81 / 0000-0504 / \$ 01.50$ 
Proposition. Let $v$ be as described in the Theorem. Then there exists a sequence of functions $\left\{g_{m}(z)\right\}_{1}^{\infty}$, each belonging to $A^{-\infty}$, such that

(a) $g_{m}(z) \neq 0(z \in U ; m=1,2, \ldots)$.

(b) $h_{m}=s g_{m}(m=1,2, \ldots)$ belong to $A^{-N}$ for some fixed $N>0$.

(c) $\left\|1-h_{m}\right\|_{-N} \rightarrow 0(m \rightarrow \infty)$.

To use this result for $A^{-p}$ we need the following

Lemma. If $g \in A^{-\infty}, g(z) \neq 0(z \in U)$, and $s g \in A^{-N}$, then $s g \in I\left(s ; A^{-N}\right)$.

Proof of the Lemma. Since $|s(z)|<1$, we have for $0<t<1$,

$$
\left|s(z)(g(z))^{t}\right| \leqslant\left|(s(z) g(z))^{t}\right| \quad \text { and thus } \quad s g^{t} \in A^{-N t} \quad(0<t \leqslant 1) .
$$

Let $F=\left\{t \mid 0 \leqslant t \leqslant 1, s g^{t} \in I\left(s ; A^{-N}\right)\right\} . F$ is closed and $0 \in F$. Let $t_{0}=\max F$. If $t_{0}<1$, choose a $\delta>0$ so that $g^{\delta} \in A^{-\left(1-t_{0}\right) N}$ and a sequence of polynomials $\left\{P_{m}\right\}_{1}^{\infty}$ so that $P_{m} \rightarrow g^{\delta}$ in $A^{-\left(1-t_{0}\right) N}$. We have $s g^{t_{0}} P_{m} \rightarrow s g^{t_{0}+\delta}$ in $A^{-N}$ and, since $s g^{t_{0}} P_{m} \in I\left(s ; A^{-N}\right)$, we obtain $s g^{t_{0}+\delta} \in I\left(s ; A^{-N}\right)$ and thus $t_{0}+\delta \in F$. Therefore $t_{0}=1$. Q.E.D.

Proof of The Theorem. Fix an arbitrary $p>0$ and show that $l \in$ $I\left(s ; A^{-p}\right)$. By the Proposition and Lemma, $1 \in I\left(s ; A^{-N}\right)$ for some $N$, i.e., $s$ is cyclic in some $A^{-N}$. Let $k>1$ be an arbitrary integer. We have $s^{1 / k} g_{m}^{1 / k} \rightarrow 1$ in $A^{-N / k}$, and hence $s g_{m}^{1 / k} \rightarrow s^{(k-1) / k}$ in $A^{-N / k}$. By the Lemma this implies $s^{(k-1) / k} \in I\left(s ; A^{-N / k}\right)$. For the same reason $s^{(k-1) / k} g_{m}^{1 / k} \rightarrow s^{(k-2) k} \in$ $I\left(s ; A^{-N / k}\right)$. After $k$ steps we obtain $1 \in I\left(s ; A^{-N / k}\right)$, and thus $s$ is cyclic in $A^{-N / k}$. Since $k$ is arbitrary, $s$ is cyclic in all $A^{-p}(p>0)$. Q.E.D.

\section{REFERENCES}

1. B. Korenblum, An extension of the Nevanlinna theory, Acta Math. 135 (1975), $187-219$.

2. H. S. Shapiro, Weakly invertible elements in certain function spaces, and generators in $l_{1}$, Michigan Math. J. 11 (1964), 161-165.

3. B. Korenblum, A Beurling-type theorem, Acta Math. 138 (1977), 265-293.

DEPARTMENT OF MATHEMATICS AND STATISTICS, STATE UNIVERSITY OF NEW YORK, ALBANY, NEW YORK 12222 